\title{
Risk Factors for Mortality in Pneumocystis jirovecii Pneumonia in Patients with Inflammatory Bowel Disease
}

\author{
Atsushi Yoshida ${ }^{a}$ Noriko Kamata $^{b}$ Akihiro Yamadac Yoko Yokoyamad Teppei Omori ${ }^{\mathrm{e}}$ \\ Toshimitsu Fujii $^{f}$ Ryohei Hayashig ${ }^{\text {Tetsu Kinjo }}{ }^{h}$ Akira Matsui ${ }^{i}$ Norimasa Fukata ${ }^{j}$ \\ Sakuma Takahashik ${ }^{k}$ Ryosuke Sakemi ${ }^{1}$ Noriyuki Ogatam Shinya Ashizukan ${ }^{\mathrm{m}}$ Shigeki Bamba ${ }^{\circ}$ \\ Makoto Ooi $^{p}$ Shuji Kanmura ${ }^{q}$ Katsuya Endor ${ }^{r}$ Takuya Yoshino $^{s}$ Hiroki Tanakat $^{t}$ \\ Toshio Morizane $^{a}$ Shinichiro Shinzaki ${ }^{\mathrm{u}}$ Taku Kobayashiv $^{\mathrm{v}}$ \\ ${ }^{a}$ Center for Gastroenterology and Inflammatory Bowel Disease, Ofuna Chuo Hospital, Kamakura, Japan; ${ }^{b}$ Department \\ of Gastroenterology, Osaka City University Graduate School of Medicine, Osaka, Japan; 'Department of Internal \\ Medicine, Sakura Medical Center, Toho University, Tokyo, Japan; ${ }^{\mathrm{d} D e p a r t m e n t}$ of Inflammatory Bowel Disease, Division \\ of Internal Medicine, Hyogo College of Medicine, Nishinomiya, Japan; ' Institute of Gastroenterology, Tokyo Women's \\ Medical University, Tokyo, Japan; ' Department of Gastroenterology and Hepatology, Tokyo Medical and Dental \\ University, Tokyo, Japan; ${ }^{9}$ Department of Endoscopy, Hiroshima University Hospital, Hiroshima, Japan; hepartment \\ of Endoscopy, University of the Ryukyus Hospital, Nishihara, Japan; 'Department of Gastroenterology, Toranomon \\ Hospital, Tokyo, Japan; 'Department of Gastroenterology and Hepatology, Kansai Medical University, Hirakata, \\ Japan; ' Department of Gastroenterology, Kagawa Prefectural Central Hospital, Takamatsu, Japan; 'Department of \\ Gastroenterology, Tobata Kyoritsu Hospital, Kitakyushu, Japan; m Digestive Disease Center, Showa University Northern \\ Yokohama Hospital, Yokohama, Japan; ${ }^{\mathrm{n} C i r c u l a t o r y}$ and Body Fluid Regulation, Faculty of Medicine, University of \\ Miyazaki, Miyazaki, Japan; ${ }^{\circ}$ Division of Clinical Nutrition, Shiga University of Medical Science, Otsu, Japan; ${ }^{\text {PDivision }}$ \\ of Gastroenterology, Department of Internal Medicine, Kobe University Graduate School of Medicine, Kobe, Japan; \\ qDepartment of Endoscopy, Kagoshima University Hospital, Kagoshima, Japan; rDepartment of Gastroenterology, \\ Tohoku Medical and Pharmaceutical University, Sendai, Japan; ${ }^{5}$ Division of Inflammatory Bowel Disease, Digestive \\ Disease Center, Kitano Hospital, Osaka, Japan; ${ }^{\mathrm{t} I B D}$ Center, Sapporo Kosei General Hospital, Sapporo, Japan; \\ "Department of Gastroenterology and Hepatology, Osaka University Graduate School of Medicine, Suita, Japan; \\ ${ }^{v}$ Center for Advanced IBD Research and Treatment, Kitasato University Kitasato Institute Hospital, Tokyo, Japan
}

\section{Keywords}

Predictive factors · Albumin · Sulfamethoxazole-

trimethoprim · Ulcerative colitis · Crohn's disease

\begin{abstract}
Background: Pneumocystis jirovecii pneumonia (PJP) is highly fatal once infection is established. In this study, we investigated the risk of PJP mortality in patients with inflammatory bowel disease (IBD). Methods: We conducted a retrospective observational study of case data from IBD patients
\end{abstract}

who developed PJP, compiled from 17 collaborating institutions. Parameters such as age, sex, medications used, and blood test results were analyzed to identify risk factors for mortality. Results: The mortality rate among the 28 IBD patients who developed PJP was $17.9 \%$. A low serum albumin level at the start of IBD treatment was identified as a risk factor for mortality and showed the following association with probability of death $(P): P=1 /[1+\exp (-5.5+2.4 \times$ Alb). The probability of death exceeded 0.5 when serum albumin was $2.2 \mathrm{~g} / \mathrm{dL}$ or lower. Conclusion: Patients with IBD who develop PJP have a high mortality rate and often cannot continue

\section{KARGER}

(C) 2019 S. Karger AG, Basel

E-Mail karger@karger.com

www.karger.com/iid
Atsushi Yoshida, MD

Center for Gastroenterology and Inflammatory Bowel Disease

Ofuna Chuo Hospital

Ofuna 6-2-24, Kamakura 247-0056 (Japan)

E-Mail atushi.y@kza.biglobe.ne.jp 
treatment with medication alone. Therefore, it is necessary to pay attention to albumin levels at the start of immunosuppressive therapy when creating a treatment plan.

(C) 2019 S. Karger AG, Basel

\section{Introduction}

It is now possible to induce and maintain remission in many patients with inflammatory bowel disease (IBD) through immunosuppressive therapy. However, reports of patients developing the opportunistic infection Pneumocystis jirovecii pneumonia (PJP) due to their immunocompromised state have also increased [1-7]. PJP is rare in the general population with an annual incidence of only $0.01-0.015 \%$, but a study showed that $14.9 \%$ of PJP patients have IBD [8], and a large cohort study revealed that the incidence of PJP among IBD patients is higher than in individuals without IBD (hazard ratio [HR] 2.96, 95\% confidence interval [CI] 1.75-4.29) [9]. Age ( $\geq 55$ years) and use of multiple immunosuppressants, such as steroids, thiopurine, and anti-tumor necrosis factor (anti-TNF) therapy, in particular have been identified as risk factors for PJP [10]. However, no previous study has investigated PJP mortality risk in IBD patients.

PJP is also known to be highly lethal once contracted. For this reason, Western consensus statements recommend primary prophylaxis with sulfamethoxazole-trimethoprim in patients on triple immunosuppressive therapy including anti-TNF therapy or a calcineurin inhibitor [7]. However, whether prophylaxis is truly necessary for all IBD patients remains under debate. We, therefore, decided to investigate PJP mortality risk in IBD patients in this study. Identification of risk factors for mortality would make it possible to avoid deaths from PJP and to limit use of sulfamethoxazole-trimethoprim prophylaxis to only those patients who require it.

\section{Materials and Methods}

About 50 institutions across Japan represented at the Achievement of IBD Total Management (AIM) Jr conference supported by EA Pharma were invited to participate, and data were collected from the 17 institutions with PJP patients who expressed intent to participate. Subjects were IBD patients who visited any of the participating institutions from January 1, 2002, to December 31, 2017. Data compiled from IBD patients who developed PJP were evaluated retrospectively in an observational study. Parameters such as age, sex, disease type, medication use history, and blood test results were evaluated.
PJP was diagnosed by either (1) the presence of characteristic features of pneumonia (e.g., interstitial pattern) on computed tomography or X-ray along with detection of elevated $\beta-\mathrm{D}$ glucan level or (2) a positive sputum polymerase chain reaction test.

History of use of immunosuppressants (e.g., anti-TNF therapy, steroids, calcineurin inhibitors, and thiopurine) to treat IBD from 2 months before onset of PJP to the outcome date after onset of PJP was analyzed. Results of blood tests performed during IBD treatment before PJP onset (up to 2 months before onset) and at PJP onset were also analyzed. The primary endpoint was PJP mortality in IBD patients. IBD outcomes after PJP onset were evaluated as a secondary endpoint.

The tenets of the Declaration of Helsinki and ethical guidelines regarding medical research on human subjects were adhered to. The study design was observational, and the protocol was approved by the ethics committees of each participating institution.

Factors contributing to mortality risk were analyzed by using Cox regression analysis and logistic regression analysis with evaluated parameters as explanatory variables. In univariate logistic regression analysis, prognosis was estimated using the following equations: $\operatorname{logit}(p)=p /(1-p)=y, p=1 /(1+\exp [-y])$. Each explanatory variable was also compared between patients who died of PJP and patients who survived using either Student's $t$ test or the $\chi^{2}$ test.

\section{Results}

Data for 28 patients with IBD who developed PJP were compiled from participating facilities. Patients were mainly male (75\% male, $25 \%$ female) and elderly (mean age \pm standard deviation $60.1 \pm 13.6$ years), and the majority had ulcerative colitis rather than Crohn's disease (85.8 vs. $14.2 \%$ ). Severity of ulcerative colitis at PJP onset based on the Mayo score for ulcerative colitis activity was moderate in almost all patients, with a mean score of 7.3 \pm 2.1 . Almost all patients $(89.3 \%)$ were on steroids to treat IBD. The next most frequently used category of drugs were immunomodulators at $60.1 \%$ (Table 1 ).

Most of the patients were using 2 (61\% of patients, 17 of 28$)$ or 3 (25\% of patients, 7 of 28$)$ immunosuppressants, but $14 \%$ (4 of 28 ) of patients developed PJP while on a single immunosuppressant. No patient who developed PJP received prophylaxis with sulfamethoxazoletrimethoprim.

In the primary endpoint analysis for mortality, PJP mortality was $17.9 \%$ (95\% CI 2.4-30.9) (Fig. 1). Mean time to death was $26.6 \pm 18.1$ days. Patients who died were significantly older than those who survived ( $69.0 \pm 5.4$ vs. $58.0 \pm 14.0$ years; $p=0.011$, Student's $t$ test) (Table 1 ).

In multivariate analysis using the Cox proportional hazards model, serum albumin at the start of IBD treatment was found to be related to mortality risk during the 2 months after PJP onset (HR 0.04, 95\% CI 0.002-0.97; $p=0.048$ ). 
Table 1. Baseline characteristics

\begin{tabular}{|c|c|c|c|c|}
\hline & $\begin{array}{l}\text { All } \\
(n=28)\end{array}$ & $\begin{array}{l}\text { Mortality } \\
\text { group } \\
(n=5)\end{array}$ & $\begin{array}{l}\text { No-mortality } \\
\text { group } \\
(n=23)\end{array}$ & $p$ value \\
\hline Sex & & & & $0.780^{*}$ \\
\hline Male & $21(75)$ & $4(80)$ & $17(74)$ & \\
\hline Female & $7(25)$ & $1(20)$ & $6(26)$ & \\
\hline Median age $\pm S D$, years & $60.1 \pm 13.6$ & $69 \pm 5.4$ & $58 \pm 14$ & $0.011^{* *}$ \\
\hline IBD type & & & & $0.690^{*}$ \\
\hline Ulcerative colitis & $24(86)$ & $4(80)$ & $20(87)$ & \\
\hline Crohn's disease & $4(14)$ & $1(20)$ & $3(13)$ & \\
\hline \multicolumn{5}{|l|}{ IBD therapy } \\
\hline Steroid & $25(90)$ & $5(100)$ & $20(87)$ & 0.390 \\
\hline Thiopurine & $17(60)$ & $4(80)$ & $13(57)$ & $0.330^{*}$ \\
\hline Calcineurin inhibitor & $7(25)$ & $2(40)$ & $5(22)$ & $0.390^{*}$ \\
\hline Anti-TNF & $10(36)$ & $0(0)$ & $10(44)$ & $0.066^{*}$ \\
\hline \multicolumn{5}{|l|}{ Laboratory data } \\
\hline Lymphocytes $\pm \mathrm{SD}, / \mu \mathrm{L}$ & $1,361 \pm 648$ & $1,077 \pm 522$ & $1,406 \pm 667$ & $0.400^{* *}$ \\
\hline Albumin $\pm S D, g / d L$ & $3.3 \pm 0.73$ & $2.6 \pm 0.81$ & $3.5 \pm 0.62$ & $0.090^{* *}$ \\
\hline C-reactive protein $\pm S D, \mathrm{mg} / \mathrm{dL}$ & $2.3 \pm 3.4$ & $2.9 \pm 2.0$ & $2.2 \pm 3.7$ & $0.560^{* *}$ \\
\hline \multicolumn{5}{|l|}{ IBD activity } \\
\hline Mayo score \pm SD & $7.3 \pm 2.1$ & & & \\
\hline Crohn's Disease Activity Index \pm SD & $310 \pm 114$ & & & \\
\hline Days \pm SD to start steroid treatment & $4.0 \pm 3.9$ & $2.2 \pm 1.3$ & $4.4 \pm 4.2$ & $0.063^{\star *}$ \\
\hline
\end{tabular}

Many patients who died were using multiple immunosuppressants, but this trend was not significant (Table 2). When the relationship between serum albumin and mortality was examined in univariate logistic regression analysis, serum albumin at the start of IBD treatment was identified as a significant risk factor for mortality (odds ratio $0.09,95 \%$ CI $0.01-0.52 ; p=0.027)$. No other factors, including age at the start of treatment, drugs used in treatment, and C-reactive protein, were found to be significant (online suppl. Table 1; see www.karger.com/doi/10.1159/000495035 for all online suppl. material). When the probability of death within 2 months of PJP onset was calculated using the results of this logistic regression analysis, the equation was $P=1 /(1+$ $\exp [-5.5+2.4 \times \mathrm{Alb}])$. This shows that the probability of death exceeded 0.5 when serum albumin was $2.2 \mathrm{~g} / \mathrm{dL}$ or lower (Fig. 2). Receiver operating characteristics curve analysis showed that a serum albumin cutoff of $2.75 \mathrm{~g} / \mathrm{dL}$ predicted PJP mortality with $80 \%$ sensitivity and $87 \%$ specificity (online suppl. Fig. 1). Total steroid dose from the start of IBD treatment to PJP onset (over the prior 2 months) was

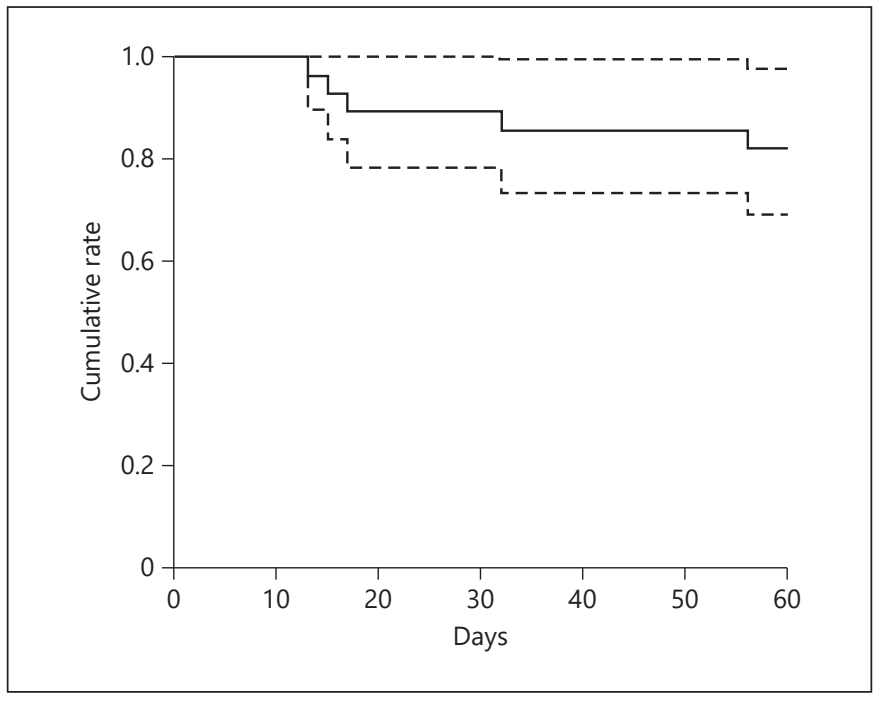

Fig. 1. Survival curve after Pneumocystis jirovecii pneumonia morbidity (the solid line is the survival curve; the dotted line is the CI). The survival rate is $82.1 \%$ (95\% CI 69.1-97.6) at 60 days. CI, confidence interval. 
Table 2. Risk factors associated with mortality (multivariate analysis using Cox proportional hazards analysis)

\begin{tabular}{lccl}
\hline & HR & $95 \%$ CI & $p$ value \\
\hline Sex & 2.12 & $0.13-33.70$ & 0.596 \\
Age & 1.02 & $0.8-1.17$ & 0.834 \\
C-reactive protein & 0.81 & $0.51-1.30$ & 0.393 \\
Number of immuno- & & & \\
$\quad$ suppressive agents & 1.38 & $0.14-13.65$ & 0.783 \\
Albumin & 0.04 & $0.002-0.97$ & 0.048 \\
\hline
\end{tabular}

$\mathrm{HR}$, hazard ratio; $\mathrm{CI}$, confidence interval.

also a significant risk factor for mortality in univariate logistic regression analysis (odds ratio 7.45, 95\% CI 1.4276.48; $p=0.040$ ).

IBD outcome analysis revealed that almost half of all patients (46.4\%), including those who died as well as the $21.4 \%$ of patients who underwent surgery and the $7.14 \%$ of patients with a serious complication requiring an intervention such as intubation (e.g., respiratory failure) or dialysis (e.g., renal failure), were ultimately unable to continue IBD treatment with medication alone (online suppl. Table 2). Therefore, it is necessary to keep in mind that IBD becomes difficult to treat with medication alone after onset of PJP. It was also possible to maintain remission in 6 of 9 patients who received repeated anti-TNF therapy combined with prophylaxis with sulfamethoxazole-trimethoprim or another drug and in 3 of 9 patients with antiTNF therapy alone.

\section{Discussion/Conclusion}

Our study was the first cohort study of PJP mortality in the field of IBD. We developed an equation for predicting PJP mortality risk based on our finding of low serum albumin levels in patients who died of PJP.

The risk of opportunistic infections is known to be high in IBD and other diseases treated with immunosuppressive therapy, and PJP has a particularly high mortality rate. Several past studies have examined PJP mortality rates in patients with IBD. In one study, the mortality rate among patients who developed PJP after starting treatment with infliximab for IBD or chronic rheumatoid arthritis was reported as high as $27 \%$ (23 of 84) [11]. In another study, the mortality rate among patients found to have PJP during treatment with infliximab for Crohn's disease was $30 \%$ (3 of 10) [12]. However, the incidence of

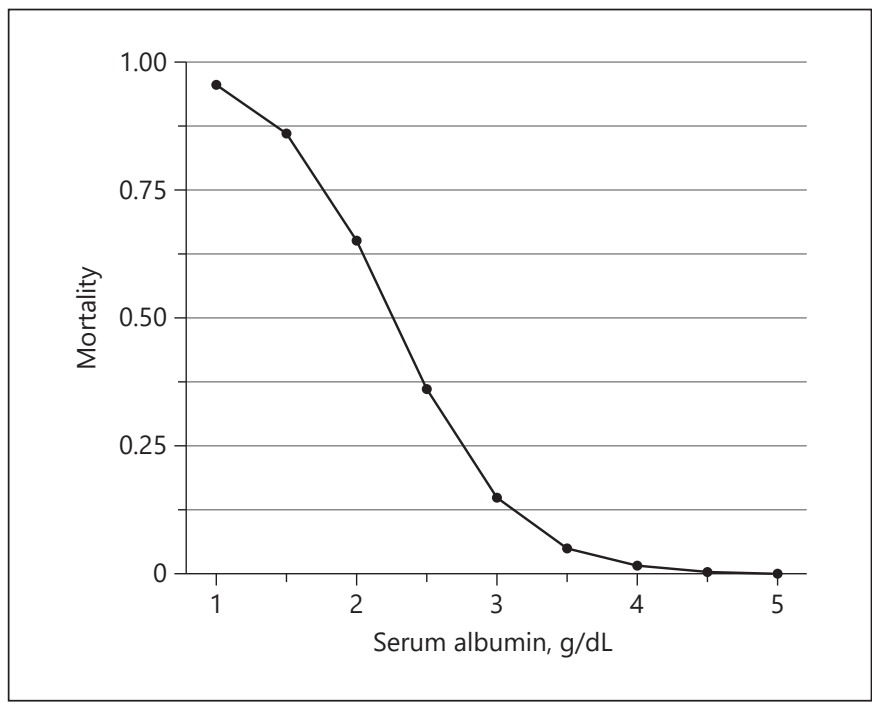

Fig. 2. Correlation between mortality and serum albumin level. The prediction formula of mortality is logit (probability) $=-5.5+2.4 \times$ Alb (g/dL), conducted by logistic regression analysis. If serum albumin level is $<2.2 \mathrm{~g} / \mathrm{dL}$ at induction therapy, the mortality rate after Pneumocystis jirovecii pneumonia onset will be $50 \%$ or more.

PJP was not reported in the phase II or III clinical trials of infliximab for Crohn's disease. This may be due to differences in patient populations and the low incidence of PJP. In a study of 86 patients with ulcerative colitis being treated with calcineurin inhibitors, the only patient developed PJP and died [13]. In summary, the reported PJP mortality is clearly high. We similarly found a high mortality rate $(17.9 \%)$ in this study, and the mean PJP mortality rate among IBD patients calculated from our results combined with results of past reports would be $25 \%$ (95\% CI $17-32 \% ; \tau^{2}=0.0051, I^{2}=0$ ) (online suppl. Table 3 ). For this reason, it is extremely important to identify risk factors for mortality after PJP onset.

Our study was the first to examine risk factors for PJP mortality in the field of IBD, but other studies have identified risk factors in patients with other underlying diseases. In a study of PJP mortality risk in patients with HIV, multivariate analysis showed that overall mortality was $13.5 \%$ and that age was related to mortality risk (adjusted odds ratio 1.54, 95\% CI 1.11-2.23; $p=0.011$ ) [14]. In this study, we did not find age to be a risk factor for mortality in Cox regression analysis or logistic regression analysis but did find in univariate analysis (Student's $t$ test) that patients who died were significantly older than those who survived. Possible reasons for this could be that our study only included IBD patients who developed PJP, the sample size was small, and the mean age of the patient 
population was higher than that of past studies. As such, age may be found to be a significant risk factor for mortality when more patients are analyzed going forward. A multivariate analysis of PJP mortality in patients who had received a kidney transplant or had chronic rheumatoid arthritis or systemic lupus erythematosus showed that development of respiratory failure and percent increase in serum KL-6 were related to 90-day mortality [15]. In a recent review article, age and development of respiratory failure, among other factors, were identified as poor prognostic factors in PJP patients without HIV infection [16]. The 5 patients who died in our study were all on mechanical ventilation within 1 week after PJP onset and had rapidly progressing respiratory failure. However, development of serious respiratory failure would be difficult to predict during treatment for IBD before PJP onset. Consequently, we hoped that identifying factors that could predict PJP mortality risk in advance would help avoid development of serious respiratory failure.

Serum albumin, which we identified as a risk factor for mortality in our study, has also been shown to be associated with mortality in studies of PJP in several other diseases. The difference in mean serum albumin between patients who died and patients who survived was -0.39 (95\% CI -0.49 to $-0.28 ; p<0.001)$, demonstrating that mean serum albumin was significantly lower in patients who died [16]. This suggests the validity of the equation for prognostic prediction that we obtained through this study.

Western consensus statements recommend primary prophylaxis with sulfamethoxazole-trimethoprim in patients on triple immunosuppressive therapy including anti-TNF therapy or a calcineurin inhibitor [7]. The main adverse reactions to sulfamethoxazole-trimethoprim are rash, gastrointestinal disorders, and hematological (hematopoietic) disorders. Rash is a relatively common reaction and manifests at varying degrees of severity from an extremely mild rash to toxic epidermal necrolysis. In a cost-effectiveness analysis of sulfamethoxazole-trimethoprim prophylaxis, a micro-simulation using a Markov model in patients with Crohn's disease at risk for PJP showed that prophylaxis for PJP was only cost-effective in patients on triple immunosuppressive therapy [17]. Therefore, whether prophylaxis with sulfamethoxazoletrimethoprim is appropriate in IBD patients on $\leq 2 \mathrm{im}$ munosuppressants remains debatable. We believe that the fact that none of the patients in our study had received prophylaxis with sulfamethoxazole-trimethoprim contributed to development of PJP in these patients. Our results also suggest that there is also a mortality risk for patients on 2 immunosuppressants, as only 1 of the 5 pa- tients who died was on triple therapy with a steroid, thiopurine, and a calcineurin inhibitor, whereas 3 were on double therapy with a steroid and thiopurine and 1 was on double therapy with a steroid and a calcineurin inhibitor. Multivariate analysis with the Cox proportional hazards model showed that the number of medications used in immunosuppressive therapy was not a risk factor for mortality (Table 2). Our finding that patients on double immunosuppressive therapy also had poor outcomes in this study indicates that prophylaxis with sulfamethoxazole-trimethoprim should be given greater consideration for all patients with low albumin.

Early detection of PJP has been identified as a favorable prognostic factor, and death can be avoided by early diagnosis [16]. However, we did not evaluate the effects of early diagnosis of PJP in this study.

None of the numerous previous studies on PJP have evaluated IBD treatment outcomes. This means that the findings of our study are novel. A particularly noteworthy finding was that a high proportion ( $78 \%$ of patients, 7 of 9) of patients who were able to continue treatment with medication alone received sulfamethoxazole-trimethoprim to enable repeated treatment with anti-TNF therapy. In addition, $21.4 \%$ of the patients required surgical treatment and all of the patients had ulcerative colitis. This indicates that avoidance of infection risk associated with immunosuppressive therapy influenced treatment selection in patients with IBD who developed PJP.

Our study has a few limitations. Firstly, we were unable to determine the incidence or risk of PJP because we did not know the total number of patients in the population. In addition, only 28 patients developed PJP, and this sample size was insufficient for multivariate analysis. Past relatively large-scale studies include a cohort study by Fillatre et al. [8]. They evaluated 293 patients with various disease conditions who developed PJP; the study was conducted using the national hospital discharge database. Another was a cohort study of 38 PJP patients conducted by Long et al. [9] using data from the LifeLink Health Plan Claims Database. However, because PJP is rare among IBD patients, we believe that our study is still a valuable cohort study examining mortality risk in 28 patients from multiple institutions. Due to the retrospective study design, we cannot rule out the influence of confounding factors on PJP mortality risk. For example, we did not investigate the influence of treatment strategy after PJP onset. Therefore, our predictive equation and other findings of this study must be validated prospectively.

In conclusion, because IBD patients who develop PJP have a high mortality rate and often cannot continue 
treatment with medication alone, it is necessary to take note of albumin levels at the start of immunosuppressive therapy when creating a treatment plan and to consider prophylaxis with sulfamethoxazole-trimethoprim.

\section{Acknowledgement}

The research team would like to sincerely thank Dr. Toshifumi Hibi, who initiated and organized the AIM Jr conference, as well as the conference attendees.

\section{Statement of Ethics}

The tenets of the Declaration of Helsinki and ethical guidelines regarding medical research on human subjects were adhered to. The study design was observational. The protocol was approved by the ethics committees of each participating institution. The study was performed in accordance with the ethics guidelines in Japan and was allowed implementation without obtaining individual written informed consent. This study was announced by posters in the outpatient ward or on the homepage of the participating institutions. Patients who expressed unwillingness to participate in this study were excluded.

\section{Disclosure Statement}

The authors have no conflicts of interest to declare.

\section{Funding Sources}

Researchers for this study were recruited at the AIM Jr Conference. The conference was funded by Eisai and EA Pharma, but the researchers did not receive direct financial support for any aspects of study design including protocol development, statistical analysis, or manuscript writing. Therefore, this study is not related to Eisai or EA Pharma.

\section{Author Contributions}

A.Yo., N.K., T.F., and T.Y. designed the study. A.Yo., N.K., A.Ya., Y.Y., T.O., T.F., R.H., T.Ki., A.M., N.F., S.T., R.S., N.O., S.A., S.B., M.O., and S.K. collected the data. A.Yo. and T.M. analyzed the data. A.Yo., N.K., A.Ya., T.F., S.B., K.E., and H.T. interpreted the data. A.Yo. drafted the manuscript, S.S. and T.Ko. made critical revisions to the manuscript. All authors reviewed and approved the final version of the manuscript.

\section{References}

1 Oshitani N, Matsumoto T, Moriyama Y, Kudoh S, Hirata K, Kuroki T. Drug-induced pneumonitis caused by sulfamethoxazole, trimethoprim during treatment of Pneumocystis carinii pneumonia in a patient with refractory ulcerative colitis. J Gastroenterol. 1998 Aug;33(4):578-81.

2 Raychaudhuri SP, Siu S. Pneumocystis carinii pneumonia in patients receiving immunosuppressive drugs for dermatological diseases. Br J Dermatol. 1999 Sep;141(3):528-30.

3 Seddik M, Meliez H, Seguy D, Viget N, Cortot A, Colombel JF. Pneumocystis jiroveci (carinii) pneumonia following initiation of infliximab and azathioprine therapy in a patient with Crohn's disease. Inflamm Bowel Dis. 2004 Jul;10(4):436-7.

4 Kasai S, Tokuda H, Otsuka Y, Ookohchi Y, Handa H, Emoto N, et al. Two cases of respiratory infection complicating treatment with infliximab. Nihon Kokyuki Gakkai Zasshi. 2007 Apr;45(4):366-71. Japanese.

5 Lee JC, Bell DC, Guinness RM, Ahmad T. Pneumocystis jiroveci pneumonia and pneumomediastinum in an anti-TNFalpha naive patient with ulcerative colitis. World J Gastroenterol. 2009 Apr;15(15):1897-900.

6 Escher M, Stange EF, Herrlinger KR. Two cases of fatal Pneumocystis jirovecii pneumonia as a complication of tacrolimus therapy in ulcerative colitis-a need for prophylaxis. J Crohns Colitis. 2010 Nov;4(5):606-9.
7 Rahier JF, Magro F, Abreu C, Armuzzi A, Ben-Horin S, Chowers Y, et al.; European Crohn's and Colitis Organisation (ECCO). Second European evidence-based consensus on the prevention, diagnosis and management of opportunistic infections in inflammatory bowel disease. J Crohns Colitis. 2014 Jun;8(6):443-468.

8 Fillatre P, Decaux O, Jouneau S, Revest M, Gacouin A, Robert-Gangneux F, et al. Incidence of Pneumocystis jiroveci pneumonia among groups at risk in HIV-negative patients. Am J Med. 2014 Dec;127(12):1242.e11-7.

9 Long MD, Farraye FA, Okafor PN, Martin C, Sandler RS, Kappelman MD. Increased risk of pneumocystis jiroveci pneumonia among patients with inflammatory bowel disease. Inflamm Bowel Dis. 2013 Apr;19(5):1018-24.

10 Okafor PN, Nunes DP, Farraye FA. Pneumocystis jiroveci pneumonia in inflammatory bowel disease: when should prophylaxis be considered? Inflamm Bowel Dis. 2013 Jul; 19(8):1764-71.

11 Kaur N, Mahl TC. Pneumocystis jiroveci (carinii) pneumonia after infliximab therapy: a review of 84 cases. Dig Dis Sci. 2007 Jun;52(6): 1481-4.
12 Velayos FS, Sandborn WJ. Pneumocystis carinii pneumonia during maintenance anti-tumor necrosis factor-alpha therapy with infliximab for Crohn's disease. Inflamm Bowel Dis. 2004 Sep;10(5):657-60.

13 Arts J, D'Haens G, Zeegers M, Van Assche G, Hiele M, D'Hoore A, et al. Long-term outcome of treatment with intravenous cyclosporin in patients with severe ulcerative colitis. Inflamm Bowel Dis. 2004 Mar; 10(2):73-8.

14 Walzer PD, Evans HE, Copas AJ, Edwards SG, Grant AD, Miller RF. Early predictors of mortality from Pneumocystis jirovecii pneumonia in HIV-infected patients: 1985-2006. Clin Infect Dis. 2008 Feb;46(4):625-33.

15 Kotani T, Katayama S, Miyazaki Y, Fukuda S, Sato Y, Ohsugi K. Risk factors for the mortality of pneumocystis jirovecii pneumonia in non-HIV patients who required mechanical ventilation: a retrospective case series study. BioMed Res Int. 2017;2017:7452604.

16 Liu Y, Su L, Jiang SJ, Qu H. Risk factors for mortality from pneumocystis carinii pneumonia (PCP) in non-HIV patients: a metaanalysis. Oncotarget. 2017 Aug;8(35):5972939.

17 Okafor PN, Farraye FA, Okafor AT, Erim DO. Cost-effectiveness of prophylaxis against pneumocystis jiroveci pneumonia in patients with Crohn's disease. Dig Dis Sci. 2015 Dec; 60(12):3743-55. 